\title{
Contents, Vol. 1, 1893/94
}

Dermatologisehe Zeitsehrift.

Herausgegeben

von

Prof. Dr. O. LASSAR.

Band I. 1893/94.

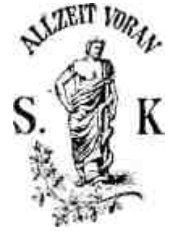

Berlin 1894

VERLAG VON S. KARGER

Charitéstrasse 3 .

$$
\mathrm{J} 1 / 8^{3} / 8^{3 / 4} 4^{1 / 8}
$$

All $\theta$ Rechte vorb $\theta$ halten.

Druck von Hans Mamroth, Berlin W., Willielmstr. 48.

lnhaltsverzeichnis.

HEFT 1.

Seite

I. Ueber den Stand und die Ziele der Dermatologie.

Yom Herausgeber.

3

IT. Zur Pathogenese der Pemphigusblasen. Von Dr.

E. Kromayer, Privatdocent für Dermatologie an der

Universität Halle. 11

III. Das Angiokeratom. Von Dr. Max Joseph in Berlin. 16

(Hierzu Tafel I.)

IV. Zur Kenntnis der hyalinen Degeneration der Car-

cinomepithelien. Von P. G. Unna. 28

(Hierzu Tafel II.)

V. Doppelseitiger Naevus verrucosus (Nervennaevus).

Von Dr. Edmund Saalfeld in Berlin. 36

(םMit einer Abbildung im Text).

VI. Ueber traumatische Epithelcysten der Finger. Von

Prof. Dr. Garrè, I. Assistenzarzt der chirurgischen Klinik

zu Tubingen. 45

VIT. Uebe'r Tuberculose des Unterhautzellgewebes. Von

Dr. E. Wermann in Dresden. 52

VIII. Experimenteller Beitrag zur Behandlung der Tu

berculose mit besonderer Berücksichtigung des 
Creosots. Von Privatdoçent Dr. med. L. Friedheim,

Assistenzarzt für Haut- und Geschlechtskrankheiten an

der Universitatspoliklinik zu Leipzig. 55

IX. Ueber die Behandlung des Lupus mit Tuberculin. Von.Dr. H. Kossel, Assistent am Institut

für Infections-krankheiten des Herrn Geheimrat Koch in Berlin. ... 79 (Hierzu Tafel III-VII.)

X. Das Verhältnis der Milchdrüse zu den Hautdrüsen.

Von Dr. C. Benda. 94

(Mit 16 Abbildungen im Text.)

BesprechungenundNotizen. Ill

IV -

HEFT 2.

Seite

XL Ueber Tuberculosis verrucosa cutis. Von Stabsarzt Dr. Heyse, Assistent der I. medicinischen Universitäts-

klinik in Berlin.

(Hierzu Tafel VIII und 2 Abbildungen im Text.)

XII. Ueber den Einfluss der Schweisssecretion auf das Blut. Von Dr. E. Grawitz, Stabsarzt und Assistent der II. medicin. Universitätsklinik in Berlin. 136

XIII. Xaevus verrucosus unius lateris (v. Bärensprung). naevus lienaris verrucosus (Unna) s.

ichtyosi-formis. Von Dr. AlfredLanz, Ordinator am Miassnitzky-

Hospital in Moskau. 142

(Hierzu Tafel X und 2 Abbildungen im Text.)

$\mathrm{X} 1 \Lambda \mathrm{r}$. Farbenanalytische Untersuchungen über gonor-rhoischen Eiter. Ein Beitrag zur Frage der eosinophilen Zellen. Von Professor Dr. C· Posner

und Dr. Arthur Leivin in Berlin. $\quad 150$

(Hierzu Tafel IX.)

XV. Ueber Cystitis colli gonorrhoica. Von Dr. Leopold

Casper, Privatdocent an der Universität Berlin. - 158 (Mit einer Abbildung im Text.)

XVI. Frische Gonorrhoe bei Frauen. VonProEDr. J. Yeit. 108

XVII. Der Zusammenbang zwischen Syphilis und Tabes dorsualis. Klínisch und kritisch

behandelt von Dr. H. Lsaac und Dr. Koch. Vorträge gebalten in der Berliner

Dermatologischenf $\Lambda^{\wedge}$ ereinigung am 4. Juni 1893.

I. Klinischer Toil. Von Dr. H. Isaac. .... 177 II. Litterarischer Teil. Von Dr. Koch. ... 181

XVIII. Kurze therapeutische Mitteilungen aus der uro-

logischen Praxis. Einige Erleichterungen beim

Katheterisieren von schwierigen Harnröhrenstricturen.

Von Dr. A. Phïlypson in Hamburg. 195

HEFT 3.

XIX. Ueber Impetigo herpetiformis. Von Dr. Johannes

Breier. (Aus der geburtshülflicben Klinik des Herrn

Geheimrat Gusserow.) 199

XX. Ueber Lungensyphilis. Von Dr. C. Schirren in Kiel. 221

XXI. Die Syphilis unter den Urvölkern Amerikas, mit besonderer Bezugnahme auf ihr Bestehen

daselbst vor der Entdeckung Amerikas durch 
Columbus. Von Prof. Dr. L. Wolff, Arzt des

deutschen Hospitals zu Philadelphia. 226

$-\mathrm{V}-$

Seite

XXII. Ueber Syphilis des äusseren Ohres. Von Or. Paul

Frey. (Aus $\mathrm{He} \prod \cdot n$ Dr. Edmund Saalfeld's Poliklinik í'iir

Hautkraiikheiten in Berlin.) 233

XXIli. Der Aussatz in Aegypten zu Moses Zeiten. Vor-trag in der ärztlichen Gesellschaft zu

Kiew am 28. November 1893 von Prof. Dr. O. N. Munch. .... 242

XXIV. Casuistische Mitteilu $\pi g e^{5} / 8$. $\lambda$ ron Dr. Ernst Feibes in Aachen.

I. Dermatitis papillomaformis circumscripta

capillitii 2o/

iil. Ueber eine oigentümliclie Art und-Anord-

nung' eines Jodex'an tliems bei einem halb-

seitig G elähm ton 201

XXV. Bin Fall von Baelz'scher Krankheit. Von Dr.

T. Broes van Dort in Rotterdam. 262

XXVI. Erythema stria turn. Von Dr. Waldemar Peter,

I. A ssistenzarzt an Prof. Dr. Lassar's Klinik für Haut-

krankheiten. 265

(iiiierzu Tafel XI.)

XXVII. Fremdkörper in der Harnrölire. Von Dr. H. Gol-

denbery in New-York.

271

XXVIII. Fremdkörper in der Blase. Nach einer Demon-. stration in der Berliner mediciniscben Gesellschaft am 24. Januar 1894 von Dr. Martin Friedländer. • $\cdot 273$

Besprechungen und Notizen. 274

HEFT 4.

XXIX. Ein Fall von Morb $\pi$ s Addisonii. (Aus dem Augusta-Hospital, zu Berlin). Von C. A.

Eivald, in Gemeinschaft $\square$ mit den Herren DDr. Mertsdiiny, Jacobson und

Jellinek bearbeitet. 279

(Hierzu Tafel XII).

XXX. Peliosis gonorrhoica und Chorea postgonorrlioica.

Vorläufige Mitteilung von Professor Dr. Litten. · 300

XXXI. Ueber faradischen u'.nd Leitungswiderstand der Haut bei Sklerodermia und eine

modificierte Methode der faradischen Widerstandsbestim-mung. Von Professor Dr. Eulenbury in

Berlin. 315

Ueber Pruritus vulvae. Von Dr. A. Cøemjín. · 324

Ueber die Perspiration bei Hautkranken. .Von

Prof. Dr. R. Quincke in Kiel. ‥ . 330

$\mathrm{t}$

$-\mathrm{VI}-$

Seite

XXXIV. Ueber das Eczem der Bindehaut und Hornhaut

des Auges. Vortrag in der Berliner ophthalmologi-

schen Gesellschaft am 15. März 1894 gehalten von

Professor Dr. Burchardt. 336 
XXXV. Ueber Pityriasis rubra und die Beziehungen

zwischen Hautkrankheiten und Pseudoleu-

kaemie. Von Dr. Waldemar Peter, I. Assistenz-

arzt an Professor Dr. Lassars Klinik für Haut

krankheiten. 345

XXXVl. Ueber die chronische G onorrhoe desMannes und

ihre Heilbarkeit. Von Dr. Martin Friedländer.

(Aus Professor Dr. Lassars Klinik) 356

XXXVII. Zur Kenntnis der syphilitischen Gelenker-

krankungen. Von Professor Dr. Renvers. Vor

trag, gehalten in der Berliner Dermatologischen Ver-

einigung am 13. Februar 1894.

XXXVIII. Die im höheren L·ebensalter erworbene Syphilis

und die Krankheiten des Nervensystems.

Von Professor Dr. E. Mendel. Vortrag gehalten

in der Berliner Dermatologischen Vereinigung am

13. Februar $1894 \quad 371$

XXXIX. Zur Diagnostik der Palmar-Affectionen. Von

Professor Dr. 0. Lassar. $\quad$ • 375

(Hierzu Tafß1 XIII-XV.)

Buch-Anzeigen. - Besprechungen und Xotizen. 379

HEFT 5.

XL. Zur Therapie der Furunkulose. Von Dr. J, Neu-

burger in Xürnberg. 387

XL·I. Ueber eine seltene Uebertragungsart der

Syphilis. Ein klinischer Beitrag von Dr. Emit

Senger in Crefeld. . . . 392

XLlt. Untersuchungen über den Partellarreflex insbesondere bei Lues. Von Dr. Marx in Berlin. 397

XLПÍ. Die hypodermatische Quecksilberbehandlung der Syphilis. Von Prof. Dr. L. Wolff in Philadelphia, üebersetzt von Dr. E. Lehfeld in Berlin. 407

XLIV. Das Eczem der Chirurgen. Vortrag auf dem

Chirurgen-Congress, von Professor Dr. 0. Lassar. 424

XLV. Xeroderma pigmentosum. Ein casuistischer Beitrag. Von Dr. F. Schütte, Assistenzarzt an Professor

Dr. Lassar's Klinik für Hautkrankheiten.

429

(Hierzu Tafel XVI.) 